\title{
Never hold the patient culpable for a bad outcome-Perhaps we were in part incorrect
}

\author{
Michael D. Black, MD, MBA
}

See related article on pages 613-23.

Neurocognitive deficits after cardiopulmonary bypass have been recognized as a serious procedural complication since the inception of heart surgery. Modifications of the pump and associated ancillary equipment have evolved; we have come a long way from disk or bubbler oxygenators and the routine use of defoaming agents. Neurologic deficits perhaps remain our, that is, the thoracic surgeon's, Achilles heel. It should therefore be no surprise that an increased attention on postoperative neurocognitive morbidity (even if the latter rates remained stable or decreased) has been seen concomitant with improved contemporary surgical mortality. Mere lack of death is no longer an acceptable procedural outcome. Having a neurocognitive postoperative deficit dramatically affects the patient's quality of life and his or her societal contributions. In addition, the fear of "postpump brain" is frequently used as a reason to search for less invasive technologies.

Surgeons are inventors, introspective and data driven, and as such we have been able to assist the medical industry in searching for plausible etiologies and treatments to deal with our own inadequacies. Examples include filtration of microemboli, plasticizers, and foreign particulate matter; tighter control of desired perfusate temperatures; and the continuous in-line surveillance of electrolyte concentration and hemodynamic parameters. Yet with all our efforts and technology, there still remain patients with less than ideal neurocognitive outcomes. Type I neurologic deficits include events such as stroke and may perhaps be easier to explain and treat. Type II deficits encompass global cognitive deficits, such as memory loss, confusion, and deterioration in global intellectual function. The latter constellation of symptoms remains more troublesome for us to explain,

\footnotetext{
From the Pediatric and Adult Congenital Heart Program, Division of Cardiac Surgery, Palm Beach Children's Hospital, and The Adult Congenital Cardiac Center at St Mary's Hospital, West Palm Beach, Fla.

Disclosures: Author has nothing to disclose with regard to commercial support.

Received for publication Oct 30, 2014; accepted for publication Oct 31, 2014; available ahead of print Nov 25, 2014.

Address for reprints: Michael D. Black, MD, MBA, Pediatric and Adult Congenital Heart Program, Division of Cardiac Surgery, Palm Beach Children's Hospital, and The Adult Congenital Cardiac Center at St Mary's Hospital, 927 45th St, Suite 301, West Palm Beach, FL 33407 (E-mail: blackm@mba.berkeley.edu).

J Thorac Cardiovasc Surg 2015;149:624-5

$0022-5223 / \$ 36.00$

Copyright (C) 2015 by The American Association for Thoracic Surgery

http://dx.doi.org/10.1016/j.jtcvs.2014.10.122
}

conceive, or combat with our current level of medical knowledge.

This article, as have others within our specialty, has refocused our attention on the patient as harboring innate genomic instructions authorizing unexpected outcomes. Although we were all taught never to hold the patient culpable for a bad outcome, perhaps we were in part incorrect. Drug bioavailability, channelopathies, and perhaps congenital cardiac malformations are easier to comprehend from a genetic perspective; but should we be less surprised that a predisposition to inflammation, T-cell activation, and subsequent neurologic injury should be any less individual? We may seem similar phenotypically, but we are after all genetically unique. If it were ethical, moral, or even permissible, a preoperative brain biopsy with cerebral tissue genetic sequencing would seem most proficient at addressing this hypothesis. But unlike with most genetic sequencing involving diseased target organs, the investigators instead resort to assessing brain dysfunction indirectly by analyzing gene expression through messenger RNA transcripts (not genes, as many transcripts examined coded for the same genes) from the peripheral circulation with a biomedical tool, the Affymetrix HG-U133 Plus 2.0 array (Affymetrix, Inc, Santa Clara, Calif). To prevent cross-hybridization, the investigators used strict filtering techniques with a previously proven high degree of reliability to give further credence to their investigations.

One obvious question: How does the peripheral blood gene expression mirror that of the brain? Fortunately, genes associated with inflammation have been well correlated across body systems (genes associated with inflammation and T-cell activation) and are currently a reliable tool in investigating neurologic degenerative disorders, such as the more common and recognized neurologic diseases Parkinson and Alzheimer. The investigators retrospectively used Ingenuity Pathway Analysis (Qiagen, Redwood City, Calif) to assist in the grouping of genes into semifunctional categories on the basis of the limitations of examining the narrow spectrum (140 or fewer) of differentially regulated genes. Although significant differences in gene expression existed postoperatively, the variances of genetic expression decreased with time, leading the investigators to conclude that "most of the genes that were differentially regulated in the neurocognitive decline group were found preoperatively." Although genes were not directly associated with one another in specific, identifiable pathways, the bioinformatics analysis revealed that the latter clusters of genes 
shared a common role in neurologic health related to overall function and cognition.

The investigators both give credence to their hypothesis that a cohort of patients remains genetically susceptible to neurocognitive injury and correctly identify multiple limitations of their experiments. Clearly, further prospective investigations will be required to substantiate the tremendous efforts identified in this study, but the article remains a good start and builds upon the investigators' previous work. The use of novel RNA sequencing, microarrays, and bioinformatics analysis will be crucial for additional elucidation of how we as human beings remain distinct from each other, with both advantages and disadvantages in how we respond to a myriad of environmental perturbations. Perhaps one day not too long in the future, routine preoperative genetic analysis will allow for a more patient-oriented, homogeneous, and successful postoperative outcome. 